\title{
Are Gamma-ray Novae Intrinsically Rare Or Just Nearby?
}

\author{
Paul J. Morris* \\ Oxford Astrophysics. Denys Wilkinson Building, Keble Road, Oxford, OX1 3RH, United \\ Kingdom \\ E-mail: paul.morrisephysics.ox.ac.uk
}

\section{Garret Cotter}

Oxford Astrophysics. Denys Wilkinson Building, Keble Road, Oxford, OX1 3RH, United

Kingdom

E-mail: garret.cotter@physics.ox.ac.uk

\section{Anthony M. Brown}

Department of Physics and Centre for Advanced Instrumentation, Durham University, Durham, DH1 3LE, United Kingdom

E-mail: anthony.brown@durham.ac.uk

\section{Paula M. Chadwick}

Department of Physics and Centre for Advanced Instrumentation, Durham University, Durham, DH1 3LE, United Kingdom

E-mail: p.m.chadwick@durham.ac.uk

Fermi LAT data revealed classical novae as unexpected gamma-ray sources, yet only 6 of 69 of those optically detected in the first 8 years of Fermi LAT observations were confirmed as $>5 \sigma$ gamma-ray sources. These proceedings outline Monte Carlo simulations in which a population of Galactic novae were simulated based on spatial distributions and R-band magnitudes based on their M31 counterparts. Interstellar extinction was added using a double exponential disc model, and gamma-ray properties were defined based on those of the original 6 gamma-ray novae. We demonstrate that observations are consistent will all classical novae being gamma-ray sources, and that the gamma-ray sky background is the largest inhibitor when discovering these sources. Furthermore, we predict that all classical novae occurring within $\approx 8 \mathrm{kpc}$ and with $m_{R} \leq 12$ will be detected using the Fermi LAT.

7th Fermi Symposium 2017

15-20 October 2017

Garmisch-Partenkirchen, Germany

\footnotetext{
* Speaker.
} 


\section{Introduction}

The progenitor system that gives rise to a classical nova $(\mathrm{CN}, \mathrm{CNe}$ plural) is a cataclysmic variable $(\mathrm{CV})$ in which a white dwarf accretes material from a lower mass main sequence star. This long term accretion can eventually result in a thermonuclear runaway (TNR) to occur on the surface of the white dwarf (e.g. 1) which observationally leads to an optical brightness increase of around 12 magnitudes (2).

Such events were not thought to produce $>100 \mathrm{MeV} \gamma$-rays, yet since the Fermi Large Area Telescope (LAT) detection of V407 Cyg in 2010 (3) a further 3 novae have been detected to $>5 \sigma$ at these high energies $(4 ; 5 ; 6 ; 7 ; 8 ; 9 ; 10 ; 11)$. The $\gamma$-ray novae all exhibit very similar high energy light curves in terms of their peak brightness and duration. Both of these features are expected to be a function of distance, yet in the first 8 years of Fermi LAT data only 6 of 69 optically discovered (12) ${ }^{1}$ novae have been confirmed as $\gamma$-ray sources.

These proceedings summarise the work contained in (13) in which populations of simulated novae were generated to determine if all $\mathrm{CNe}$ being $\gamma$-ray sources is consistent with observations.

\section{Methodology}

We base our simulated population of $\mathrm{CNe}$ on those detected in M31, and leave the nova rate as a free parameter consistent with $\dot{N}_{\text {novae }}=35 \pm 11$ year $^{-1}$ (14). CNe data from 1909 can be obtained online $\left((15)^{2},(16)\right.$ and (17) and the references therein). M31 was divided into 6 elliptical bins with 2 for the bulge and 4 for the disc. The bulge/disc boundary and bulge axis ratio was defined using isophotes from (18), whilst the disc axis ratio was derived from the inclination angle of Andromeda, $i=12.5^{\circ}$ (19), with the maximum set as $a=4.0^{\circ}$. The semi-minor axes of the disc bins were taken as $b=a \cos i$. A plot of the binning is available in (13), and was used to assign simulated $\mathrm{CNe} 2 \mathrm{D}$ coordinates in the xy plane.

R-band data was plentiful in the M31 CNe sample, and was chosen on which to base the absolute magnitudes of the simulated $\mathrm{CNe}$. After removing recurrent novae from the data, a total of 262 M31 CNe remained. Interstellar extinction was corrected using the NASA/IPAC Extragalactic Database $^{3}$ value of $A_{B}=0.300$ magnitudes which is based on HI column densities (20) and the absolute magnitude obtained by assuming an M31 distance of $780 \mathrm{kpc}$. The data were binned separately for disc and bulge novae.

A 2-sample KS test yielded a $26.5 \%$ chance that the disc and bulge populations are intrinsically the same, hence the absolute magnitudes of the simulated novae were assigned separately depending on whether located in the disc or bulge. The probability distributions for the M31 novae can be found in (13).

The simulated Milky Way was defined to have $R_{d}=20 \mathrm{kpc}$ and $a_{b}=3.0 \mathrm{kpc}$, where $d$ and $b$ denote the disc and bulge. The bulge axis ratios were set as $2: 1$ with angle $\phi=20^{\circ}$ between $a_{b}=3.0$ and the vector defined by the line from the Galactic centre to the Solar System located 8 kpc away (21).

\footnotetext{
${ }^{1}$ Accessible at http://asd.gsfc.nasa.gov/Koji.Mukai/novae/novae.html

${ }^{2}$ Accessible at: http://www.mpe.mpg.de/ m31novae/opt/m31/index.php

${ }^{3}$ https://ned.ipac.caltech.edu
} 
The $\mathrm{z}$ positions for disc $\mathrm{CNe}$ were assumed to follow an exponential disc profile (22), whilst the bulge novae had a Gaussian distribution which was found to replicate the observed population with a $94.9 \%$ chance of deriving from the same population as the observed Galactic novae. Other functions were tested in (13).

It was assumed that interstellar extinction in the Milky Way was consistent with the double exponential dust distribution model of (22), which was integrated along the line of sight to each nova in order to redden the R-band magnitude.

As a $\mathrm{CNe}$ is most likely to be detected in $\gamma$-rays at peak brightness, $\gamma$-ray luminosities were assigned to simulated $\mathrm{CNe}$ assuming a flat distribution between the peak one day values of the original $6 \gamma$-ray novae.

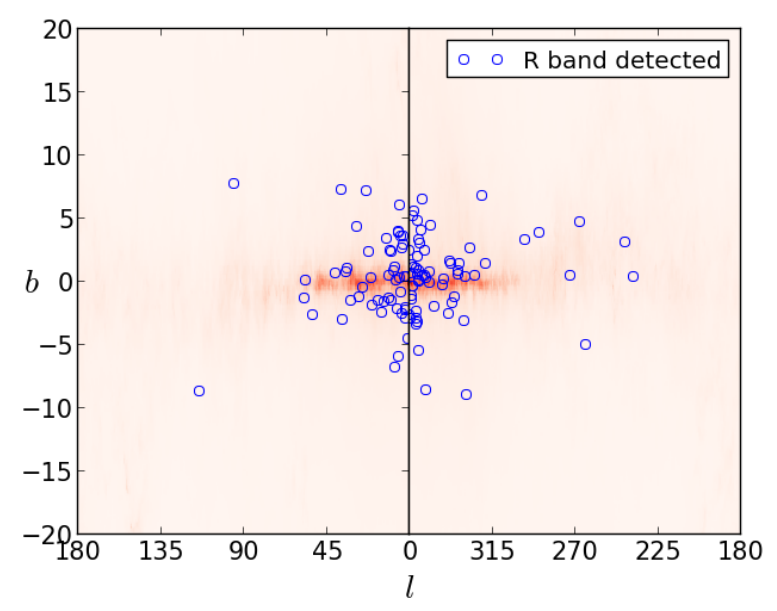

(a) Simulated Novae

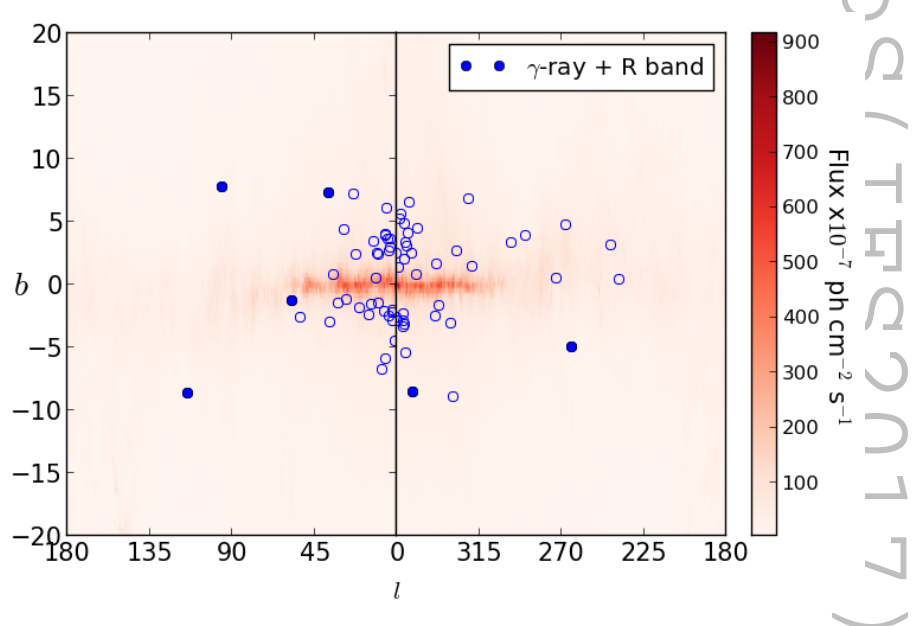

(b) Visible Novae

Figure 1: (a) Example simulated population. (b) Visible simulated $\mathrm{CNe}$ accounting for interstellar extinction. The colour scale represents the intensity of the $\gamma$-ray sky background.

R-band discovery of a simulated CNe was if $m_{R}<m_{t h}$, for some threshold magnitude $m_{t h}$, which was left as a free parameter. A nova was only considered a $\gamma$-ray source if the $\gamma$-ray flux exceeded the mean ratio of the detected novae to the background flux from the Galactic diffuse, $F_{\text {GalDiff }} . \gamma$-ray novae were those that met the criteria for both $\gamma$-ray detection and R-band detection.

\section{Results}

Fig. 1 illustrates that our rudimentary model can easily reproduce an observed nova distribution which closely resembles the actual Milky Way distribution. Such a result implies that the Milky Way nova population is consistent with being bulge dominated.

Fig. 2 demonstrates that the number of $\gamma$-ray discoverable novae is independent of the threshold R band magnitude, $m_{t h}$, for all reasonable $m_{t h}$ values. It can be seen that even for a relatively bright $m_{t h}$, very few novae are discovered in $\gamma$-rays alone.

Fig. 3 shows that the $\gamma$-ray detected nova, V5856 Sgr (23), which has occurred since this study was made, lies within our predicted parameter space. 


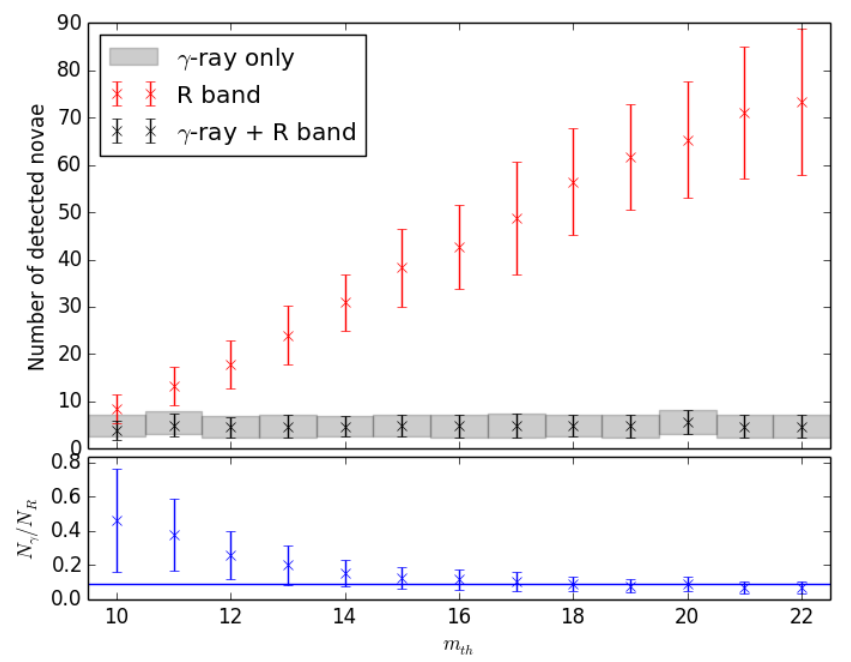

Figure 2: Figure taken from (13) showing how the number of R-band and $\gamma$-ray detected novae vary as a function of $m_{t h}$. Error bars are the standard deviations from 100 runs of the code.

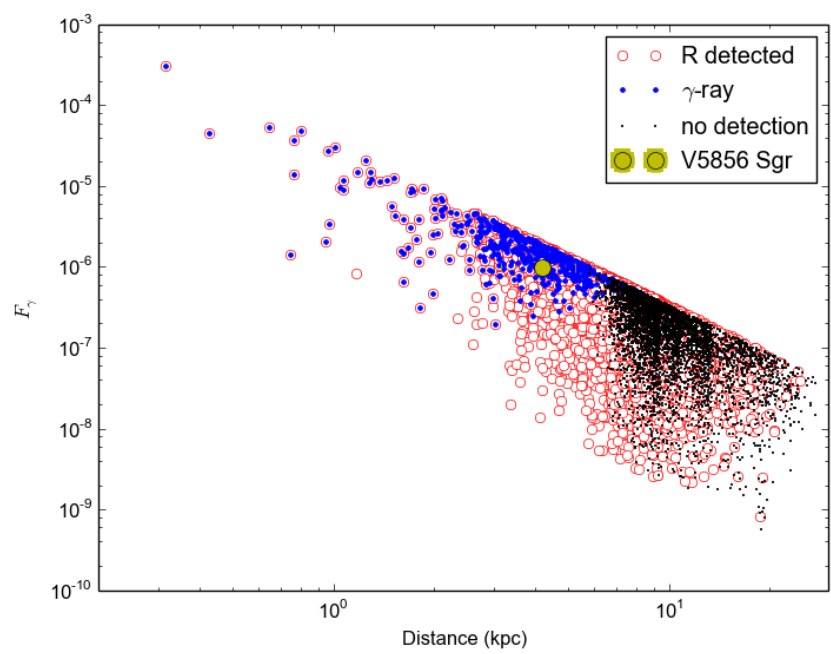

Figure 3: Figure showing the observed $\gamma$-ray peak flux as a function of distance to the CNe. V5856 Sgr, a $\mathrm{CNe}$ which has occurred since this study was made, lies in the expected parameter space.

\section{Discussion}

Fig. 1 shows that the simple model presented here is capable of reproducing the distribution of $\mathrm{CNe}$ on the sky. It is apparent from the figure that novae detected in $\gamma$-rays tend to lie at least a few degrees from the Galactic plane. This can be explained as nearby $\mathrm{CNe}$ have a greater chance to be detected at higher $|l|$, and higher $|l|$ regions suffer from less interstellar extinction and typically have less extreme $\gamma$-ray background fluxes so are more likely to be confirmed as $\gamma$-ray novae. Thus we expect $\gamma$-rays to be emitted from all $\mathrm{CNe}$ and those we detect are simply are nearby subset of the whole population.

Fig. 2 demonstrates that the total number of $\gamma$-ray detectable $\mathrm{CNe}$ for which there exists an 
optical counterpart, as $N_{\gamma}$ is independent of $m_{R, t h}$ and is consistent with being constant. Such a result implies that the limiting factor inhibiting the discovery of more $\gamma$-ray is the $\gamma$-ray sky background rather than the effects of interstellar extinction. Furthermore, $\mathrm{CNe}$ are unlikely to be unidentified $\gamma$-ray sources.

Fig. 3 shows that the parameter space in which we expect $\gamma$-ray novae to be discovered is for $d \leq 8 \mathrm{kpc}$, with the majority of these within $6 \mathrm{kpc}$. By ignoring off plane effects, we therefore expect to be able to observe $\approx(6 \mathrm{kpc} / 20 \mathrm{kpc})^{2}=9 \%$ using the Fermi LAT. A similar figure in (13) also shows we expect $\mathrm{CNe}$ with $m_{R}<12$ to also be confirmed as $\gamma$-ray sources, further restricting our parameter space. Since the publication of (13), it can be seen that the $>5 \sigma \gamma$-ray confirmed $\mathrm{CNe}$ V5856 Sgr lies in our predicted region, indicating our conclusions are sound.

\section{Conclusion}

We have shown that observations of $\mathrm{CNe}$ in $>100 \mathrm{MeV} \gamma$-rays made using the Fermi LAT are consistent with the entire $\mathrm{CNe}$ population being $\gamma$-ray sources. Furthermore, we have demonstrated that the $\gamma$-ray sky background is the main inhibitor to detection more of these sources at these wavelengths. We predict that all $\mathrm{CNe}$ occurring within $\approx 8 \mathrm{kpc}$ will be detected as $\gamma$-ray sources.

\section{Acknowledgements}

This work was supported by the Oxford Centre for Astrophysical Surveys which is funded through generous support from the Hintze Family Charitable Foundation. GC acknowledges support from STFC grants ST/N000919/1 and ST/M00757X/1 and from Exeter College, Oxford.

\section{References}

[1] M. M. Shara, Recent progress in understanding the eruptions of classical novae, Publ.Astron.Soc.Pacific. 101 (Jan., 1989) 5-31.

[2] B. W. Carroll and D. A. Ostlie, An Introduction to Modern Astrophysics and Cosmology. Pearson, San Francisco, $2^{\text {nd }}$ ed., July, 2006.

[3] A. A. Abdo, M. Ackermann, M. Ajello, W. B. Atwood, L. Baldini, J. Ballet et al., Gamma-Ray Emission Concurrent with the Nova in the Symbiotic Binary V407 Cygni, Science 329 (Aug., 2010) 817-821, [1008.3912].

[4] C. C. Cheung, T. Glanzman and A. B. Hill, Fermi LAT Detection of a New Galactic Bulge Gamma-ray Transient in the Scorpius Region: Fermi J1750-3243, and its Possible Association with Nova Sco 2012, The Astronomer's Telegram 4284 (July, 2012) .

[5] C. C. Cheung, S. N. Shore, I. De Gennaro Aquino, S. Charbonnel, J. Edlin, E. Hays et al., Possible Association of the Gamma-ray Transient Fermi J0639+0548 with Nova Mon 2012, The Astronomer's Telegram 4310 (Aug., 2012) .

[6] E. Hays, T. Cheung and S. Ciprini, Detection of gamma rays from Nova Delphini 2013, The Astronomer's Telegram 5302 (Aug., 2013) . 
[7] C. C. Cheung, P. Jean and S. N. Shore, Fermi-LAT Observations of Nova V1369 Centauri 2013 Brightening in Gamma rays, The Astronomer's Telegram 5653 (Dec., 2013) .

[8] C. C. Cheung, P. Jean, F. L. A. T. Collaboration and S. N. Shore, Fermi-LAT Gamma-ray Observations of Nova Sagittarii 2015 No. 2, The Astronomer's Telegram 7283 (Mar., 2015) .

[9] K.-L. Li and L. Chomiuk, Fermi-LAT detection of the Galactic nova TCP J18102829-2729590, The Astronomer's Telegram 9699 (Nov., 2016) .

[10] C. C. Cheung, P. Jean, S. N. Shore and Fermi Large Area Telescope Collaboration, Fermi-LAT Gamma-ray Observations of Nova Lupus 2016 (ASASSN-16kt), The Astronomer's Telegram 9594 (Oct., 2016) .

[11] K.-L. Li, L. Chomiuk and J. Strader, Fermi-LAT detection of a very bright Gamma-ray Onset from the Galactic Nova ASASSN-16ma, The Astronomer's Telegram 9736 (Nov., 2016) .

[12] K. Mukai, Koji's list of recent galactic novae, 2016.

[13] P. J. Morris, G. Cotter, A. M. Brown and P. M. Chadwick, Gamma-ray novae: rare or nearby?, MNRAS 465 (Feb., 2017) 1218-1226, [1610 . 09941 ].

[14] A. W. Shafter, On the Nova Rate in the Galaxy, ApJ 487 (Sept., 1997) 226-236.

[15] Max-Planck-Institut für extraterrestrische Physik, M31 (apparent) optical nova catalogue,, 2015.

[16] W. Pietsch, F. Haberl, G. Sala, H. Stiele, K. Hornoch, A. Riffeser et al., X-ray monitoring of optical novae in M 31 from July 2004 to February 2005, A\&A 465 (Apr., 2007) 375-392, [astro-ph/0612596].

[17] W. Pietsch, X-ray emission from optical novae in M 31, Astronomische Nachrichten 331 (Feb., 2010) 187, [0910.3865].

[18] R. L. Beaton, S. R. Majewski, P. Guhathakurta, M. F. Skrutskie, R. M. Cutri, J. Good et al., Unveiling the Boxy Bulge and Bar of the Andromeda Spiral Galaxy, ApJL 658 (Apr., 2007) L91-L94, [astro-ph/0605239].

[19] F. Simien, A. Pellet, G. Monnet, E. Athanassoula, A. Maucherat and G. Courtes, The spiral structure of M31 - A morphological approach, A\&A 67 (June, 1978) 73-79.

[20] D. Burstein and C. Heiles, Reddenings derived from H I and galaxy counts - Accuracy and maps, The Astronomical Journal 87 (Aug., 1982) 1165-1189.

[21] J. Binney, O. Gerhard and D. Spergel, The photometric structure of the inner Galaxy, MNRAS 288 (June, 1997) 365-374, [astro-ph/9609066].

[22] P. C. Dawson and R. G. Johnson, The Visibility of Galactic Supernovae, JRASC 88 (Dec., 1994) 369.

[23] K.-L. Li, B. D. Metzger, L. Chomiuk, I. Vurm, J. Strader, T. Finzell et al., A nova outburst powered by shocks, Nature Astronomy 1 (Oct., 2017) 697-702, [1709.00763]. 